CIRJE-F-960

\title{
Connected Price Dynamics with \\ Revealed Preferences and Auctioneer's Discretion in VCG Combinatorial Auction
}

\author{
Hitoshi Matsushima \\ The University of Tokyo
}

February 2015

CIRJE Discussion Papers can be downloaded without charge from:

http://www.cirje.e.u-tokyo.ac.jp/research/03research02dp.html

Discussion Papers are a series of manuscripts in their draft form. They are not intended for circulation or distribution except as indicated by the author. For that reason Discussion Papers may not be reproduced or distributed without the written consent of the author. 


\title{
Connected Price Dynamics with Revealed Preferences and Auctioneer's Discretion in VCG Combinatorial Auction*
}

\author{
Hitoshi Matsushima** $^{* *}$ \\ Department of Economics, University of Tokyo
}

November 23, 2010

This Version: February 26, 2015

\begin{abstract}
We investigate a general class of dynamical open-bid combinatorial auction protocols termed price-demand procedures, where the auctioneer asks buyer-dependent price vectors and buyers reveal demand sets. Such revelations are easier to practice than the revelations of entire valuations at once. With revealed preference activity rule and connectedness, we characterize the class of all procedures that achieve the VCG outcome correctly. Since buyers can detect whether the auctioneer succeeds to achieve the VCG outcome just by observing the history, they can leave the selection of procedure the auctioneer's discretion. The auctioneer can save irrelevant information leakage by selecting a signal-contingent shortcut.
\end{abstract}

Keywords: Combinatorial Auctions, Price-Demand Procedures, VCG Mechanisms, Revealed Preference Activity Rule, Connectedness, Representative Valuation Functions.

JEL Classification Numbers: D44, D47, D61, D82, D86

\footnotetext{
* This paper is a substantial revision of my earlier work (Matsushima (2011)). It reports the findings of a study that was supported by a grant-in-aid for scientific research (KAKENHI 21330043) from the Japan Society for the Promotion of Science (JSPS) and the Ministry of Education, Culture, Sports, Science and Technology (MEXT) of the Japanese government. I am grateful to the co-editor and an anonymous referee for their useful comments. All errors are mine.

** Department of Economics, University of Tokyo, Hongo, Bunkyo-ku, Tokyo 113-0033, Japan. E-mail: hitoshi[at]e.u-tokyo.ac.jp
} 


\section{Introduction}

This paper investigates a general class of dynamical open-bid combinatorial auction protocols termed price-demand procedures for allocating heterogeneous commodities in the efficient manner. In the continuous time horizon, the auctioneer (seller) continues to ask a price vector for all packages of commodities to each buyer (participants), and this buyer reveals a set of packages as his (or her) demand set. We permit the auctioneer to ask different price vectors across buyers. The auctioneer eventually stop asking price vectors within a time limit, and, based on the resultant history of asked price vectors and revealed demand sets, the auctioneer determines the allocation of commodities and the side payments from the buyers to the auctioneer. Hence, we define a price-demand procedure as a combination of a pricing rule and an ending rule that are history-contingent.

The purpose of this paper is to clarify the possibility that a price-demand procedure achieves the VCG (Vickery-Clarke-Groves ${ }^{1}$ ) outcome (allocation and side payments), i.e., achieve efficiency under the constraints of strategy-proofness, where each buyer is willing to reveal all the best-response packages associated with his true valuation function at all times as a dominant strategy.

We make the following two assumptions on price-demand procedures, i.e., the revealed preference activity rule and the connectedness. The revealed preference activity rule implies that each buyer is required to reveal demand sets in the manner that there exists a single valuation function, associated with which, his revealed demand set is always the same as the set of all the best responses through time. With this rule, we can replace strategy-proofness with the requirement for each buyer to conform to his true valuation function through time as a dominant strategy.

The connectedness implies that the auctioneer never makes his (or her) asked price vector jump to any price vector that he has never asked before. With this connectedness, from the observation of the history, the auctioneer can always calculate the difference in valuation for any buyer between any pair of packages that this buyer revealed in this history.

\footnotetext{
${ }^{1}$ See Vickery (1961), Clarke (1971), and Groves (1973).
} 
With the revealed preference activity rule and the connectedness, we can demonstrate a characterization result for the class of all price-demand procedures that always achieves the correct VCG outcome in the following manner. At any time, we can uniquely specify a valuation function, termed representative valuation function, for each buyer in the history-contingent manner; it assigns to any revealed package the minimal relative valuations, and assigns to any unrevealed package the maximal absolute value. In this case, any revealed package must be a best response for the representative valuation function.

Based on this specification, we show as this paper's main theorem that a price-demand procedure achieves the correct VCG outcome if and only if the efficient allocation and the efficient allocations without a single buyer associated with the profile of representative valuation functions that we specify at the ending time are all revealed in the resultant history. Hence, all the auctioneer has to do for the achievement of the correct VCG outcome is to continue to ask price vectors until he recognize that the efficient allocation and the efficient allocations without a single buyer associated with the resultant profile of representative valuation functions are all revealed in the history.

It is not a difficult task for the auctioneer to find out such a profile of representative valuation functions. In fact, the auctioneer can do this manner just by ascending the price for any revealed packages and descending the price for any unrevealed packages. Importantly, we can show that the efficient allocation and the VCG payments associated with the profile of representative valuation functions that we specify at the ending time is just the same as the efficient allocation and the VCG payments associated with the true profile of valuation functions. Hence, any buyer can easily detect whether the auctioneer succeeds to achieve the correct VCG outcome just by observing the resultant history.

Based on the above arguments, it is sufficient for the achievement of the correct VCG outcome that the auctioneer makes the following 'detail-free' contractual agreement with the buyers:

(i) The auctioneer continues to ask a price vector to each buyer under the constraint of connectedness, and requires each buyer to reveal a demand set under the constraint of revealed preference activity rule. 
(ii) This procedure continues until the history occurs that reveals the efficient allocation and the efficient allocations without any single buyer associated with the history-contingent profile of representative valuation functions.

(iii) The auctioneer eventually ends the procedure within a time limit, and then achieves the VCG outcome associated with the resultant profile of representative valuation functions.

Note that the buyers do not need to know the detail of price-demand procedure that the auctioneer follows; provided the auctioneer is sufficiently penalized whenever he fails to satisfy these requirements, the above-mentioned contractual agreement can sufficiently incentivize the auctioneer to select a price-demand procedure that achieves the correct VCG outcome.

Because of the detail-free nature, the buyers can leave the selection of price-demand procedure the auctioneer's discretion. In this case, the auctioneer can make the selection of price-demand procedure even contingent on his private signal. This signal could include non-trivial information regarding the buyers' valuation functions, but does not need to be verifiable to the court.

It is often that a buyer is afraid that any information about his valuation function, which is not necessarily relevant to the allocation problem, is leaked to the public. The auctioneer can preserve such a buyer's privacy concern by selecting a shortcut procedure. The extent to which the information regarding a buyer's valuation function is leaked to the public is well described by the resultant representative valuation function as well as the set of all revealed packages. The auctioneer can save such an irrelevant information leakage by utilizing his private information and stopping the procedure as soon as he recognize that the efficient allocation and the efficient allocations without any single buyer are all revealed in the history.

As the previous works such as Rothkopf, Teisberg, and Kahn (1990) have pointed out $^{2}$, the standard practice of the VCG mechanism, wherein buyers directly announce the entire valuations for the enormous number of packages, has flaws in practice. In fact, it is generally too complicated for them to assess and report the entire valuations and then compute the VCG outcome at one time.

${ }^{2}$ See also Milgrom (2004), Ausubel and Milgrom (2006), Parkes (2006), and Rothkopf (2007). 
Hence, it is important to search for the possibility of replacing such a standard practice with a dynamical price adjustment such as a price-demand procedure, because the sequential revelation of all the best responses to asked price vectors through time would be inherently much easier to make than the revelation of the entire valuations at once. For example, Ausubel $(2004,2006)$ demonstrated an open-bid protocol termed Ausubel mechanism or its variants as the replacement of the standard practice. Chen and Takeuchi (2010) conducted laboratory experiments and showed the better performance of an open-bid protocol termed iBEA auction than the standard practice. The present paper demonstrates, not only such easier revelation practices, but also a concrete and tractable method for deriving the VCG outcome from the resultant history.

There exist many other relevant works such as Gul and Stacchetti (2000), which examined their respective open-bid version of the VCG mechanism ${ }^{3}$. Among them, Parkes and Ungar (2002), Lahaie, Constantin, and Parkes (2005), Lahaie and Parkes (2004), and Mishra and Parkes (2007) introduced an involved market equilibrium notion termed universal competitive equilibrium for understanding such practical issues. The universal competitive equilibrium is defined as a buyer-dependent price vector that is not only a competitive equilibrium but also a competitive equilibrium without any single buyer. We can derive the efficient allocation and the VCG payments from a universal competitive equilibrium. Because of its mathematical form, we can regard a profile of representative valuation functions as a buyer-dependent price vector. We can show that the profile of representative valuation functions that we specify at the ending time is a universal competitive equilibrium for the true profile of valuation functions.

Rothkopf (2007) argued about buyers' privacy concern, and pointed out the difficulty of hiding the revealed inputs by using cryptography. This paper assumes that all such inputs are automatically leaked to the public. With this assumption, the auctioneer's careful selection of a shortcut procedure becomes quite substantial.

The remainder of this paper is organized as follows: Section 2 models the combinatorial auction problem. Section 3 introduces price-demand procedure and the

\footnotetext{
${ }^{3}$ See also Kelso and Crawford (1982), Bikhchandani and Ostroy (2002), Ausubel, Cramton, and Milgrom (2006), Parkes and Ungar (2002), Lahaie and Parkes (2004), Lahaie, Constantin, and Parkes (2005), Mishra and Parkes (2007), and others.
} 
assumption of revealed preference activity rule. Section 4 introduces the assumption of connectedness. Section 5 introduces representative valuation function and shows the characterization result. Section 6 clarifies the relationship between representative valuation function and universal competitive equilibrium. Section 7 explains the detail-free contractual agreement and the auctioneer's discretion. Section 8 explains the auctioneer's signal-contingent selection of price-demand procedure. Section 9 argues the issue on information leakage. Section 10 concludes. 


\section{The Model}

Let us investigate an combinatorial auction problem wherein $l \geq 1$ multiple heterogeneous items are traded altogether. Let $L \equiv\{1, \ldots, l\}$ denote the set of all items. The set of all buyers is denoted by $N \equiv\{1, \ldots, n\}$, where $n \geq 2$. An allocation is defined as $a \equiv\left(a_{1}, \ldots, a_{n}\right)$, where $a_{i} \subset L$ implies the package of items that is assigned to buyer $i$, and $a_{i} \cap a_{j}=\phi$ for $j \neq i$. Let $A$ denote the set of all allocations. An allocation without buyer $i \in N$ is defined as $a^{i} \equiv\left(a_{j}\right)_{j \in N \backslash\{i\}}$, where $a_{j} \subset L$ and $a_{j} \cap a_{h}=\phi$ for $h \neq j$. Let $A^{i}$ denote the set of all allocations without buyer $i \in N$.

We assume quasi-linearity. A valuation function for buyer $i \in N$ is denoted by $u_{i}: 2^{L} \rightarrow R_{+}$, where $u_{i}(\phi)=0$, and

$$
u_{i}\left(a_{i}\right)>u_{i}\left(\tilde{a}_{i}\right) \text { if } \tilde{a}_{i} \neq a_{i} \text { and } \tilde{a}_{i} \subset a_{i} .
$$

Let $U_{i}$ denote the set of all valuation functions for buyer $i$. Let $U \equiv \prod_{i \in N} U_{i}$ and $U_{-i} \equiv \prod_{j \in N \backslash\{i\}} U_{j}$

An allocation $a \in A$ is said to be efficient for a profile of valuation functions $u \in U$ if

$$
\sum_{i \in N} u_{i}\left(a_{i}\right) \geq \sum_{i \in N} u_{i}\left(\tilde{a}_{i}\right) \text { for all } \tilde{a} \in A \text {. }
$$

Let $A^{*}(u) \subset A$ denote the set of all efficient allocations for $u \in U$. An allocation $a^{i} \in A^{i}$ without buyer $i$ is said to be efficient for a profile of valuation functions without buyer $i \in N, u_{-i}=\left(u_{j}\right)_{j \in N \backslash\{i\}} \in U_{-i}$, if

$$
\sum_{j \in N \backslash\{i\}} u_{j}\left(a_{i}\right) \geq \sum_{j \in N \backslash\{i\}} u_{j}\left(\tilde{a}_{i}\right) \text { for all } \tilde{a}^{i} \in A^{i} .
$$

Let $A^{i^{*}}\left(u_{-i}\right) \subset A^{i}$ denote the set of all efficient allocations without buyer $i$ for $u_{-i} \in U_{-i} \cdot$

A direct mechanism, hereinafter a mechanism, is defined as $G=(f, x)$, where $f: U \rightarrow A$ denotes an allocation rule, and $x: U \rightarrow R^{n}$ denotes a payment rule. Let 
$f(u)=\left(f_{i}(u)\right)_{i \in N} \in A, \quad x=\left(x_{i}\right)_{i \in N}, \quad x_{i}: U \rightarrow R, \quad$ and $\quad x(u)=\left(x_{i}(u)\right)_{i \in N} \in R^{n} . \quad A$ mechanism $G$ is said to be efficient if

$$
f(u) \in A^{*}(u) \text { for all } u \in U \text {. }
$$

A mechanism $G=(f, x)$ is said to be VCG (Vickery-Clarke-Groves) if it is efficient and

$$
x_{i}(u)=\max _{a^{i} \in A^{i}} \sum_{j \in N \backslash\{i\}} u_{j}\left(a_{j}\right)-\sum_{j \in N \backslash\{i\}} u_{j}\left(f_{j}(u)\right) \text { for all } i \in N \text { and } u \in U \text {. }
$$

Note that a VCG mechanism $G=(f, x)$ is strategy-proof in that truth-telling is a dominant strategy, i.e., for every $i \in N, u \in U$, and $\tilde{u}_{i} \in U_{i}$,

$$
u_{i}\left(f_{i}(u)\right)-x_{i}(u) \geq u_{i}\left(f_{i}\left(\tilde{u}_{i}, u_{-i}\right)\right)-x_{i}\left(\tilde{u}_{i}, u_{-i}\right) .
$$




\section{Price-Demand Procedures and Revealed Preference Activity Rule}

A price vector for buyer $i \in N$ is denoted by $p_{i}=\left(p_{i}\left(a_{i}\right)\right)_{a_{i} \subset L} \in R^{2^{L}}$, where $p_{i}(\phi)=0$, and

$$
p_{i}\left(a_{i}\right)>p_{i}\left(\tilde{a}_{i}\right) \text { if } a_{i} \neq \tilde{a}_{i} \text { and } \tilde{a}_{i} \subset a_{i} .
$$

Let $P_{i}$ denote the set of all price vectors for buyer $i$. Let $P \equiv \prod_{i \in N} P_{i}$ and $p \equiv\left(p_{i}\right)_{i \in N} \in P$.

In the continuous time horizon $[0, \infty)$, we consider an open-bid protocol termed a price-demand procedure as follows ${ }^{4}$. At the beginning of the initial time 0 , the auctioneer observes a private signal $\omega$. The set of possible private signals is denoted by $\Omega$. Contingent on the observed signal $\omega$, the auctioneer selects a price-demand procedure denoted by

$$
\left(\gamma^{\omega}, T^{\omega}\right)=(\gamma, T),
$$

wherein $\gamma=\left(\gamma_{i}\right)_{i \in N}$ denotes a pricing rule and $T: U \rightarrow[0, \infty)$ denotes an ending rule, which will be defined later on.

Until Section 8, we will not explicitly take into account the signal-contingence of price-demand procedure. Hence, from this section to Section 7, we will simply denote by $(\gamma, T)$ a price-demand procedure, instead of $\left(\gamma^{\omega}, T^{\omega}\right)_{\omega \in \Omega}$.

At any time $t \in[0, \infty)$, the auctioneer asks a price vector $p_{i}=p_{i}(t) \in P_{i}$ to each buyer $i \in N$ and requires this buyer to reveal a set of packages $m_{i}=m_{i}(t) \subset 2^{L}$ as his

\footnotetext{
${ }^{4}$ This paper depends on the assumption of continuous time horizon. We may expect to replace this assumption with the discrete time horizon, without violating the substance of this paper. In this case, however, we need to assume the presence of a positive price grid, weaken the incentive requirements, and even abandon the exact achievement of the VCG outcome to a certain extent.
} 
demand set ${ }^{5}$. We permit $m_{i}$ to include the null package $\phi$, while we do not permit $m_{i}$ to be empty, i.e., $m_{i} \in 2^{2^{L}} \backslash\{\phi\}$.

The auctioneer is permitted to ask different price vectors across buyers. A combination of the asked price vector and the revealed demand set $\left(p_{i}, m_{i}\right) \in P_{i} \times 2^{2^{L}} \backslash\{\phi\}$ is said to be consistent with a valuation function $u_{i} \in U_{i}$ for buyer $i$ if $m_{i}$ is the set of all best response packages to $p_{i}$ for $u_{i}$, i.e.,

$$
m_{i}=\underset{a_{i} \in 2^{L}}{\arg \max }\left\{u_{i}\left(a_{i}\right)-p_{i}\left(a_{i}\right)\right\} .
$$

A history for buyer $i \in N$ up to time $t \in(0, \infty)$ is denoted by

$$
h_{i}^{t}:[0, t) \rightarrow P_{i} \times 2^{2^{L}} \backslash\{\phi\},
$$

where $h_{i}^{t}(\tau)=\left(p_{i}(\tau), m_{i}(\tau)\right)$. A history $h_{i}^{t}$ is said to be consistent with $u_{i} \in U_{i}$ if $h_{i}^{t}(\tau)$ is consistent with $u_{i}$ for all $\tau \in[0, t)$. Let $h_{i}^{0}$ denote the null history. Let $H_{i}^{t}\left(u_{i}\right)$ denote the set of all histories for buyer $i$ up to time $t$ that is consistent with $u_{i}$. Let

$$
\begin{aligned}
& H_{i}^{t} \equiv \bigcup_{u_{i} \in U_{i}} H_{i}^{t}\left(u_{i}\right), H^{t} \equiv \prod_{i \in N} H_{i}^{t}, H \equiv \bigcup_{t \in[0, \infty)} H^{t}, h^{t}=\left(h_{i}^{t}\right)_{i \in N} \in H^{t} \text {, and } \\
& H_{i}^{0} \equiv\left\{h_{i}^{0}\right\} .
\end{aligned}
$$

For every $h_{i}^{t} \in H_{i}^{t}$, let us define the set of all valuation functions for buyer $i$ with which $h_{i}^{t}$ is consistent, by

$$
U_{i}\left(h_{i}^{t}\right) \equiv\left\{u_{i} \in U_{i} \mid h_{i}^{t} \in H_{i}^{t}\left(u_{i}\right)\right\} .
$$

For every $h_{i}^{t} \in H_{i}^{t}$, we define the set of all packages that buyer $i$ reveals in the history $h_{i}^{t}$ by

$$
A_{i}\left(h_{i}^{t}\right) \equiv\left\{a_{i} \in 2^{L} \mid a_{i} \in m_{i}(\tau) \text { for some } \tau \in[0, t)\right\} .
$$

Let $U\left(h^{t}\right) \equiv \prod_{i \in N} U_{i}\left(h_{i}^{t}\right), \quad A\left(h^{t}\right) \equiv \prod_{i \in N} A_{i}\left(h_{i}^{t}\right)$, and $A^{i}\left(h^{t}\right) \equiv \prod_{j \in N \backslash\{i\}} A_{j}\left(h_{j}^{t}\right)$.

A pricing rule $\gamma=\left(\gamma_{i}\right)_{i \in N}$ is defined by

\footnotetext{
${ }^{5}$ We will denote $p_{i}\left(a_{i}\right)=p_{i}(t)\left(a_{i}\right)$, where $p_{i}=p_{i}(t)$.
} 


$$
\gamma_{i}: \bigcup_{t=0}^{\infty} H^{t} \rightarrow P_{i} \text { for all } i \in N
$$

At any time $t \in[0, \infty)$, where the history $h^{t} \in H^{t}$ has occurred, the auctioneer asks the price vector $\gamma_{i}\left(h^{t}\right) \in P_{i}$ to each buyer $i \in N$. Let $h^{t}=h^{t}(u, \gamma)$ denote the history up to time $t$ that occurs when the buyers continue to reveal their demand sets in the consistent manner with $u$ :

$$
h^{t}(u, \gamma) \in H^{t}(u)
$$

and

$$
p_{i}(\tau)=\gamma_{i}\left(h^{\tau}\right) \text { for all } i \in N \text { and } \tau \in[0, t),
$$

where we denote $h^{t}(u, \gamma)=\left(h_{i}^{t}(u, \gamma)\right)_{i \in N}$, and

$$
h_{i}^{t}(u, \gamma)(\tau)=h_{i}^{t}(\tau)=\left(p_{i}(\tau), m_{i}(\tau)\right) \text { for all } i \in N \text { and } \tau \in[0, t) .
$$

Importantly, this paper will assume the revealed preference activity rule, which requires each buyer to reveal the demand sets in the consistent manner with a single valuation function; for any buyer $i$, at any time $t$, the occurred history $h_{i}^{t}$ must satisfy that $U_{i}\left(h_{i}^{t}\right)$ is non-empty, i.e.,

$$
U_{i}\left(h_{i}^{t}\right) \neq \phi .
$$

With this rule, we can describe any observation of a buyer's behavior as the consequence of his selection of a valuation function as a proxy, associated with which, he continues to reveal the set of all best response packages for this selected valuation function through time.

Provided the buyers confirm to a profile of valuation functions $u \in U$ in the above-mentioned manner, the auctioneer stops asking price vectors at the ending time given by $t=T(u) \in[0, \infty)$. Importantly, we will assume that for every $u \in U\left(h^{t}\right)$ and $u^{\prime} \in U\left(h^{t}\right)$,

$$
\left[T\left(u^{\prime}\right)=T(u)\right] \Leftrightarrow\left[u^{\prime} \in U\left(h^{T(u)}(u, \gamma)\right)\right]
$$

This is a necessary assumption, because the auctioneer cannot directly make the selection of ending time contingent on the true profile of valuation functions; whenever $u^{\prime} \in U\left(h^{T(u)}(u, \gamma)\right)$, the resultant history must be the same between $u$ and $u^{\prime}$ : $h^{T(u)}(u, \gamma)=h^{T(u)}\left(u^{\prime}, \gamma\right)$ and $T\left(u^{\prime}\right)=T(u)$. We define the set of all possible histories as 


$$
H(\gamma, T) \equiv\left\{h^{t} \in H \mid h^{t}=h^{T(u)}(u, \gamma) \text { for some } u \in U\right\} .
$$

A mechanism $G=(f, x)$ is said to be consistent with a price-demand procedure $(\gamma, T)$ if for every $h^{t} \in H(\gamma, T), u \in U\left(h^{t}\right)$, and $u^{\prime} \in U\left(h^{t}\right)$,

$$
(f(u), x(u))=\left(f\left(u^{\prime}\right), x\left(u^{\prime}\right)\right) .
$$

Whenever the mechanism $G$ is consistent with the price-demand procedure $(\gamma, T)$, then the auctioneer can gather the sufficient information regarding the profile of valuation functions for achieving the outcome, i.e., the allocation and the payment vector, induced by $G$.

The following lemma shows that whenever a mechanism is efficient and consistent with a price-demand procedure, the allocation induced by this mechanism must be revealed in the resultant history.

Lemma 1: If a mechanism $G=(f, x)$ is efficient and consistent with a price-demand procedure $(\gamma, T)$, then, for every $u \in U, f(u)$ is revealed in the resultant history, i.e.,

$$
f(u) \in A\left(h^{T(u)}(u, \gamma)\right) .
$$

Proof: See Appendix A.

In order to understand this lemma, suppose that $f_{i}(u)=\phi$, and it was not revealed. Then, without loss of generality, we can assume that the valuation for the null package $\phi$, i.e., $u_{i}\left(f_{i}(u)\right)=u_{i}(\phi)$, is very small compared with any non-null package. This, however, implies that any efficient allocation never assigns buyer $i$ the null package $\phi$. This is a contradiction. Next, suppose that $f_{i}(u) \neq \phi$, and it was not revealed. Then, without loss of generality, we can assume that there is a proper subset $a_{i} \subset f_{i}(u)$ such that $u_{i}\left(f_{i}(u)\right)$ is greater than but very close to $u_{i}\left(a_{i}\right)$. In this case, we can improve the welfare by assigning buyer $i a_{i}$ instead of $f_{i}(u)$, and assigning some buyer $j \neq i$ $a_{j} \cup\left\{f_{i}(u) \backslash a_{i}\right\}$ instead of $a_{j}$. This is a contradiction. Hence, $f_{i}(u)$ must be revealed. 


\section{Connectedness}

A history $h_{i}^{t}$ for buyer $i$ up to time $t$ is said to be connected if for every $\tau \in(0, t)$,

either $p_{i}(\tau)=\lim _{\tau^{\prime} \uparrow \tau} p_{i}\left(\tau^{\prime}\right)$, or $p_{i}(\tau)=p_{i}\left(\tau^{\prime}\right)$ for some $\tau^{\prime} \in(0, \tau)$.

The connectedness implies that the auctioneer never makes his asked price vector jump to any price vector that he has never asked before. Because of this connectedness, from the observation of the history, the auctioneer can calculate the difference in valuation for any buyer between any pair of packages whenever this buyer revealed these packages in the history.

Lemma 2: For every connected history $h_{i}^{t} \in H_{i}^{t}$ and $\left\{a_{i}, a_{i}^{\prime}\right\} \subset A_{i}\left(h_{i}^{t}\right)$, there uniquely exists $x_{i}\left(a_{i}, a_{i}^{\prime}, h_{i}^{t}\right) \in R$ such that

$$
x_{i}\left(a_{i}, a_{i}^{\prime}, h_{i}^{t}\right)=u_{i}\left(a_{i}\right)-u_{i}\left(a_{i}^{\prime}\right) \text { for all } u_{i} \in U_{i}\left(h_{i}^{t}\right) .
$$

Proof: See Appendix B.

A price-demand procedure $(\gamma, T)$ is said to be connected if for every $t \in(0, \infty)$, $u \in U$, and $i \in N, h_{i}^{t}(u, \gamma) \in H_{i}^{t}$ is connected. The following proposition shows a necessary and sufficient condition for the existence of a VCG mechanism that is consistent with a connected price-demand procedure; it is necessary and sufficient that, associated with any profile of valuation functions, the efficient allocations with and without any single buyer are all revealed in the history.

Proposition 3: There exists a VCG mechanism $G$ that is consistent with a connected price-demand procedure $(\gamma, T)$ if and only if for every $h^{t} \in H(\gamma, T)$, there exist $a^{*}\left(h^{t}\right) \in A\left(h^{t}\right)$, and $a^{i *}\left(h_{-i}^{t}\right) \in A^{i}\left(h_{-i}^{t}\right)$ for each $i \in N$, such that for every $u \in U\left(h^{t}\right)$,

$$
a^{*}\left(h^{t}\right) \in A^{*}(u),
$$

and 
(4)

$$
a^{i^{*}}\left(h_{-i}^{t}\right) \in A^{i^{*}}\left(u_{-i}\right) \text { for all } i \in N
$$

Proof: See Appendix C.

If the efficient allocations with and without any buyer are all revealed in the history, it follows from Lemma 2 that the difference in valuation between the efficient allocation and the efficient allocation without any single buyer is uniquely determined from the observation of this history. This guarantees that there exists a VCG mechanism that is consistent with the price-demand procedure, because the VCG payment for each buyer is equivalent to the sum of the other buyers' differences in valuation between the efficient allocation and the efficient allocation without buyer $i$.

On the other hand, if either the efficient allocations or the efficient allocation without a buyer is unrevealed, the history fails to determine the VCG payments that are common to all the profiles of valuation functions that are consistent with this history. 


\section{Representative Valuation Functions}

For every $i \in N, \quad t \in[0, \infty)$, and connected history $h_{i}^{t} \in H_{i}^{t}$, we define the representative valuation function $u_{i}^{\left[h_{i}^{t}\right]} \in U_{i}$ as follows. Assume $u_{i}^{\left[h_{i}^{t}\right]}(\phi)=0$. Fix an arbitrary package that belongs to $A_{i}\left(h_{i}^{t}\right)$, denoted by $\tilde{a}_{i} \in A_{i}\left(h_{i}^{t}\right)$. For every $a_{i} \in A_{i}\left(h_{i}^{t}\right) \backslash\left\{\tilde{a}_{i}\right\}$, let us specify

$$
u_{i}^{\left[h_{i}^{t}\right]}\left(a_{i}\right) \equiv u_{i}^{\left[h_{i}^{t}\right]}\left(\tilde{a}_{i}\right)-x_{i}\left(\tilde{a}_{i}, a_{i}, h_{i}^{t}\right),
$$

and for every $a_{i} \notin A_{i}\left(h_{i}^{t}\right)$,

$$
u_{i}^{\left[h_{i}^{t}\right]}\left(a_{i}\right) \equiv \inf _{\tau \in[0, t), a_{i}^{\prime} \in m_{i}(\tau)}\left\{u_{i}^{\left[h_{i}^{t}\right]}\left(a_{i}^{\prime}\right)-p_{i}(\tau)\left(a_{i}^{\prime}\right)+p_{i}(\tau)\left(a_{i}\right)\right\} .
$$

The representative valuation function assigns the maximal absolute value to any unrevealed non-null package in the consistent manner with the history. It is clear by definition that the representative valuation function $u_{i}^{\left[h_{i}^{t}\right]}$ exists uniquely. Let $u^{\left[h^{t}\right]}=\left(u_{i}^{\left[h_{i}^{t}\right]}\right)_{i \in N}$ denote the profile of representative valuation functions associated with the connected history $h_{i}^{t} \in H_{i}^{t}$. The following proposition shows that the representative valuation function $u_{i}^{\left[h_{i}^{t}\right]}$ assigns any revealed package $a_{i} \in A_{i}\left(h_{i}^{t}\right)$ with the minimal possible valuation in relative terms.

Proposition 4: For every $t \in[0, \infty)$, connected history $h_{i}^{t} \in H_{i}^{t}$, and $u_{i} \in U_{i}$, it holds that $u_{i} \in U_{i}\left(h_{i}^{t}\right)$ if and only if for every $a_{i} \in A_{i}\left(h_{i}^{t}\right)$,

$$
u_{i}\left(a_{i}\right)-u_{i}\left(a_{i}^{\prime}\right)=u_{i}^{\left[h_{i}^{t}\right]}\left(a_{i}\right)-u_{i}^{\left[h_{i}^{t}\right]}\left(a_{i}^{\prime}\right) \text { for all } a_{i}^{\prime} \in A_{i}\left(h_{i}^{t}\right) \text {, }
$$

and

$$
u_{i}\left(a_{i}\right)-u_{i}\left(a_{i}^{\prime}\right)>u_{i}^{\left[h_{i}^{t}\right]}\left(a_{i}\right)-u_{i}^{\left[h_{i}^{t}\right]}\left(a_{i}^{\prime}\right) \text { for all } a_{i}^{\prime} \notin A_{i}\left(h_{i}^{t}\right) .
$$

In this case, for every $a_{i} \in A_{i}\left(h_{i}^{t}\right)$,

$$
u_{i}\left(a_{i}\right) \geq u_{i}^{\left[h_{i}^{t}\right]}\left(a_{i}\right),
$$

and

$$
u_{i}\left(a_{i}\right)=u_{i}^{\left[h_{i}^{t}\right]}\left(a_{i}\right) \text { if and only if } \phi \in A_{i}\left(h_{i}^{t}\right) .
$$


Proof: See Appendix D.

The following theorem shows that the necessary and sufficient condition in Proposition 3 can be replaced with another condition implying that associated with the profile of representative valuation functions, the efficient allocations with and without any single buyer are all revealed in the history. Hence, all we have to do for evaluating the sufficiency is to examine just the representative valuation functions.

Theorem 5: There exists a VCG mechanism $G$ that is consistent with a connected price-demand procedure $(\gamma, T)$ if and only if for every $h^{t} \in H(\gamma, T)$,

$$
A\left(h^{t}\right) \cap A^{*}\left(u^{\left[h^{t}\right]}\right) \neq \phi,
$$

and

$$
A^{i}\left(h_{-i}^{t}\right) \bigcap A^{i *}\left(u_{-i}^{\left[h_{-i}^{t}\right]}\right) \neq \phi \text { for all } i \in N
$$

Proof: See Appendix E.

By following the connected price-demand procedure $(\gamma, T)$ satisfying (5) and (6), the auctioneer can achieve a VCG mechanism $(f, x)$, which is specified as follows; for every $u \in U$,

$$
f(u) \in A^{*}\left(u^{\left[h^{T(u)}(u, \gamma)\right]}\right) \bigcap A\left(h^{T(u)}(u, \gamma)\right),
$$

and

$$
x_{i}(u)=\max _{a^{i} \in A^{i}} \sum_{j \in N \backslash\{i\}} u_{j}^{\left[h^{T(u)}(u, \gamma)\right]}\left(a_{j}\right)-\sum_{j \in N \backslash\{i\}} u_{j}^{\left[h^{T(u)}(u, \gamma)\right]}\left(f_{j}(u)\right) \text { for all } i \in N .
$$

Since the profile of representative valuation functions $u^{\left[h^{t}\right]}$ minimizes the differences in valuation between the efficient allocations and other allocations, the requirements of efficiency for $u^{\left[h^{t}\right]}$ would be the severest among all the profiles of valuation functions included in $U\left(h^{t}\right)$. Hence, it is sufficient to examine just the profile of representative valuation functions $u^{\left[h^{t}\right]}$. 


\section{Universal Competitive Equilibrium}

A profile of price vectors $p \equiv\left(p_{i}\right)_{i \in N} \in P$ is said to be a competitive equilibrium for $u \in U$ if there exists an allocation, denoted by $a^{C E}(u) \in A$, that maximizes the payoffs for the seller and the buyers, i.e.,

$$
\sum_{i \in N} p_{i}\left(a_{i}^{C E}\right) \geq \sum_{i \in N} p_{i}\left(a_{i}\right) \text { for all } a \in A,
$$

and for every $i \in N$ and $a_{i} \in A_{i}$,

$$
u_{i}\left(a_{i}^{C E}\right)-p_{i}\left(a_{i}^{C E}\right) \geq u_{i}\left(a_{i}\right)-p_{i}\left(a_{i}\right) .
$$

A profile of price vectors $p \in P$ is said to be a competitive equilibrium without buyer $i$ for $u_{-i} \in U_{-i}$ if there exists an allocation without buyer $i$, denoted by $a^{i, C E}(u) \in A^{i}$, that maximizes the payoffs for the sellers and the buyers except for buyer $i$ satisfying that

$$
\sum_{j \in N \backslash\{i\}} p_{j}\left(a_{j}^{i, C E}\right) \geq \sum_{j \in N \backslash\{i\}} p_{j}\left(a_{j}\right) \text { for all } a^{i} \in A^{i},
$$

and for every $j \in N \backslash\{i\}$ and $a_{j} \in A_{j}$,

$$
u_{j}\left(a_{j}^{i, C E}\right)-p_{j}\left(a_{j}^{i, C E}\right) \geq u_{j}\left(a_{j}\right)-p_{j}\left(a_{j}\right) .
$$

According to the same manner as the previous works such as Parkes and Ungar (2002), Lahaie and Parkes (2004), Lahaie, Constantin, and Parkes (2005), and Mishra and Parkes (2007), we define a universal competitive equilibrium for $u \in U$ as a profile of price vectors $p \in P$ such that it is a competitive equilibrium for $u$, and also for every $i \in N$, it is a competitive equilibrium without buyer $i$ for $u_{-i}$. We must note that whenever $p$ is a universal competitive equilibrium for $u$, then the allocations $a^{C E}(u)$ and $a_{-i}^{i, C E}(u)$ could satisfy the efficiency in the manner that

$$
a^{C E}(u) \in A^{*}(u) \text {, and } a^{i, C E}(u) \in A^{j^{*}}\left(u_{-i}\right) \text { for all } i \in N .
$$

Because of (1) and (2), we can express the representative valuation function by a $\left|A_{i}\right|$-dimensional vector $u_{i}^{\left[h_{i}^{t}\right]}=\left(u_{i}^{\left[h_{i}^{t}\right]}\left(a_{i}\right)\right)_{a_{i} \in A_{i}} \in R^{\left|A_{i}\right|}$, which could be regarded as a price vector for buyer $i$, i.e.,

$$
u_{i}^{\left[h_{i}^{t}\right]} \in P_{i} .
$$


The following proposition states that the profile of representative valuation functions associated with the history at the ending time is a universal competitive equilibrium.

Proposition 6: For every $h^{t} \in H^{t}$, if properties (5) and (6) are satisfied, then the profile of representative valuation functions $u_{i}^{\left[h_{i}^{t}\right]}$ is a universal competitive equilibrium for all $u \in U\left(h_{i}^{t}\right)$.

Proof: See Appendix F.

Clearly, there exists a VCG mechanism that is consistent with the following connected price-demand procedure; the auctioneer starts with declining the price vector for each buyer to a price vector that is sufficiently close to the zero vector. The auctioneer then adjusts the price vector for each buyer in the ascending manner that whenever the buyer reveals any package at the current time, then the auctioneer increases the price for this package. In this manner, any buyer eventually comes to reveal all packages, including the null package, at one time, implying that we can identify all relative valuations at one time, achieving the correct VCG outcome. In this case, the profile of price vectors at the ending time corresponds to a universal competitive equilibrium associated with the true profile of valuation functions.

The auctioneer can replace the above procedure with a shortcut procedure; the auctioneer can stop asking price vectors as soon as he recognizes that the efficient allocations with and without any single buyer associated with the profile of representative valuation functions were all revealed in the resultant history. This is relevant to the issue about the extent to which the information about the true profile of valuation functions is leaked to the public through the procedure, the detail of which will be discussed in Section 8 . 


\section{Detail-Free Contractual Agreements}

Based on the arguments in the previous sections, we can consider the situation in which the auctioneer (the seller) makes a contractual agreement with the participants (the buyers) as the bundle of the following requirements.

(i) The auctioneer continues to ask a price vector to each buyer under the constraint of connectedness, and require each buyer to reveal a demand set under the constraint of the revealed preference activity rule.

(ii) This procedure continues until the history $h^{t}$ occurs that reveals the efficient allocations with and without any single buyer associated with the profile of representative valuation functions $u^{\left[h^{t}\right]}$, i.e.,

$$
A\left(h^{t}\right) \cap A^{*}\left(u^{\left[h^{t}\right]}\right) \neq \phi,
$$

and

$$
A^{i}\left(h_{-i}^{t}\right) \bigcap A^{i *}\left(u_{-i}^{\left[h_{-i}^{t}\right]}\right) \neq \phi \text { for all } i \in N,
$$

where $t$ denotes the ending time.

(iii) The auctioneer eventually ends the procedure within a time limit, and then achieves the VCG outcome associated with the profile of representative valuation functions at the ending time $u^{\left[h^{t}\right]}$, i.e., determines $(a, s) \in A \times R^{n}$, satisfying that

$$
a \in A\left(h^{t}\right) \cap A^{*}\left(u^{\left[h^{t}\right]}\right),
$$

and

$$
S_{i}=\max _{\tilde{a}^{i} \in A^{i}} \sum_{j \in N \backslash\{i\}} u_{j}^{\left[h^{t}\right]}\left(\tilde{a}_{j}^{i}\right)-\sum_{j \in N \backslash\{i\}} u_{j}^{\left[h^{t}\right]}\left(a_{j}^{i}\right) \text { for all } i \in N,
$$

where $t$ denotes the ending time, $h^{t}$ denotes the resultant history, and $s=\left(s_{i}\right)_{i \in N}$.

With the revealed preference activity rule, we can describe any observation of a buyer's behavior as the consequence of his selection of a proxy valuation function, associated with which, he continues to reveal the set of all best response packages. This property, joint with the achievement of the VCG outcomes in the manner of (iii), can incentivize the buyers to select their true valuation functions as dominant strategies, revealing the set of all best response packages associated with their true valuation functions. 
It is not a difficult task for the auctioneer to follow these entire requirements. For example, as shown at the bottom of Section 6, the auctioneer can easily design a connected price-demand procedure with which a VCG mechanism is consistent.

Under the above-mentioned contractual agreement, the buyers can leave the selection of price-demand procedure the auctioneer's discretion. In this case, note that the buyers and the third parties such as the courts can easily detect whether the auctioneer actually follows the entire requirements, just by observing the occurred history of asked price vectors and revealed demand sets; the buyers do not need to know the detail of the price-demand procedure that the auctioneer follows. Hence, we can say that their contractual agreement could be 'detail-free'.

Provided the auctioneer is sufficiently penalized whenever he does not follow these requirements, the contractual agreement in the above manner can sufficiently incentivize the auctioneer to select a connected price-demand procedure, with which, a VCG mechanism is consistent. 


\section{Signal-Contingent Price-Demand Procedure}

According to the success of the detail-free contractual agreement explained in Section 7, the auctioneer can make the selection of a price-demand procedure even contingent on his private signal. This signal could include non-trivial information regarding the buyers' valuation functions. Importantly, this signal does not need to be verifiable to the buyers and the court. Even if the signal is informative, the auctioneer (the seller) cannot utilize it for his self-interested purpose such as his revenue maximization. The buyer can detect that the auctioneer selected an inefficient allocation just by observing the history, and then can sufficiently penalize the auctioneer.

We define a signal-contingent price-demand procedure by

$$
\Gamma \equiv\left(\gamma^{\omega}, T^{\omega}\right)_{\omega \in \Omega} .
$$

A mechanism $G=(f, x)$ is said to be consistent with a signal-contingent price-demand procedure $\Gamma$ if it is consistent with $\left(\gamma^{\omega}, T^{\omega}\right)$ for all $\omega \in \Omega$.

Let us consider a mapping $\xi: U \rightarrow \Omega$; we assume that the auctioneer observes the private signal given by $\omega=\xi(u) \in \Omega$ when $u$ is the true profile of valuation functions. By observing this signal, the auctioneer recognizes that the profile of valuation functions belongs to the set $\xi^{-1}(\omega) \subset U$, and then selects the corresponding price-demand procedure $\left(\gamma^{\omega}, T^{\omega}\right)$.

As the extreme case, let us consider the situation wherein the auctioneer observes the complete information regarding the profile of valuation functions:

$$
\Omega=U \text {, and } \xi(u)=u \text { for all } u \in U \text {. }
$$

The auctioneer can select the following connected price-demand procedure $\lambda(\omega)=(\gamma, T)$ that is consistent with a VCG mechanism. At the initial time 0 , the auctioneer asks the profile of price vectors that corresponds to the universal competitive equilibrium associated with $u=\xi^{-1}(\omega)$. The resultant ending time $T(u)$ could be the initial time 0 ; the auctioneer can immediately verify the correct efficient allocations with and without any single buyer, achieving a VCG outcome at the initial time 0. 


\section{Information Leakage}

We can regard the set of all valuation functions for each buyer $i \in N$ with which the connected history $h_{i}^{t}$ up to time $t$ is consistent, i.e., $U_{i}\left(h_{i}^{t}\right)$, as describing the extent to which the information regarding buyer $i$ 's valuation function is leaked to the public. Proposition 4 implies that $U_{i}\left(h_{i}^{t}\right)$, i.e., the extent of information leakage for buyer i's valuation functions, can be uniquely identified from the representative valuation function $u_{i}^{\left[h_{i}^{t}\right]}$ for buyer $i$ and the set of all packages that buyer $i$ reveals, $A_{i}\left(h_{i}^{t}\right)$; the combination of $u_{i}^{\left[h_{i}^{t}\right]}$ and $A_{i}\left(h_{i}^{t}\right)$ is the sufficient statistics for the extent to which the information about buyer i's valuation function was leaked to the public.

The auctioneer can preserve the buyers' privacy concern by selecting a shortcut price-demand procedure that decreases the extent of information leakage as much as possible. For example, let us consider a case of two buyers and two items, i.e., buyer 1, buyer 2, item A, and item B, in which, the profile of valuation functions $u$ is given by

$$
\begin{aligned}
& u_{1}(\{A\})=u_{2}(\{B\})=W>1, \\
& u_{1}(\{B\})=u_{2}(\{A\})=1,
\end{aligned}
$$

and

$$
u_{1}(\{A, B\})=u_{2}(\{A, B\})=100+W .
$$

We assume that the auctioneer knows the above-mentioned form of valuation functions, but does not know the value of $W \in(1, \infty)$. Note that the sets of all efficient allocations with and without any single buyer are as follows. If $W>100$, then

$$
A^{*}(u)=\{(\{A\},\{B\})\}, A^{2^{*}}\left(u_{1}\right)=\{\{A, B\}\} \text {, and } A^{1^{*}}\left(u_{2}\right)=\{\{A, B\}\} .
$$

If $1<W<100$, then

$$
\begin{aligned}
& A^{*}(u)=\{(\{A, B\}, \phi),(\phi,\{A, B\})\}, \\
& A^{2^{*}}\left(u_{1}\right)=\{\{A, B\}\}, \text { and } A^{1^{*}}\left(u_{2}\right)=\{\{A, B\}\} .
\end{aligned}
$$

If $W=100$, then

$$
\begin{aligned}
& A^{*}(u)=\{(\{A\},\{B\}),(\{A, B\}, \phi),(\phi,\{A, B\})\}, \\
& A^{2^{*}}\left(u_{1}\right)=\{\{A, B\}\}, \text { and } A^{1^{*}}\left(u_{2}\right)=\{\{A, B\}\} .
\end{aligned}
$$


We investigate the following connected price-demand procedure. At the initial time 0 , the auctioneer asks the profile of price vectors $p=\left(p_{1}, p_{2}\right)$ given by

$$
p_{1}(\{A\}\}=p_{1}(\{B\})=p_{2}(\{A\}\}=p_{2}(\{B\})=100,
$$

and

$$
p_{1}(\{A, B\}\}=p_{2}(\{A, B\})=200 .
$$

Note that this profile $p$ corresponds to the profile of representative valuation functions at the initial time 0 .

Case 1: $W>100$.

At the initial time 0 , each buyer $i$ is willing to reveal the set of all best response packages $m_{i}$ given by

$$
m_{1}=\{\{A\},\{A, B\}\} \text { and } m_{2}=\{\{B\},\{A, B\}\} .
$$

The efficient allocation $(\{A\},\{B\})$, the efficient allocation without buyer $2\{A, B\}$, and the efficient allocation without buyer $1\{A, B\}$, which are associated with the profile of representative valuation functions $p$ at the initial time 0 , were all revealed at the initial time 0 . Hence, the auctioneer can achieve the VCG outcome at the initial time 0 :

$$
\begin{aligned}
& f(u)=(\{A\},\{B\}), \\
& x_{1}(u)=p_{2}(\{A, B\})-p_{2}(\{B\})=100, \text { and } \\
& x_{2}(u)=p_{1}(\{A, B\})-p_{1}(\{A\})=100 .
\end{aligned}
$$

The price-demand procedure ends at the initial time 0 , leaking to the public just the following information:

1) Each buyer's valuation for $\{A, B\}$ is greater than or equals 200.

2) Buyer 1's valuation for $\{A\}$ is greater than that $\{B\}$. Buyer 2's valuation for $\{B\}$ is greater than that $\{A\}$.

3) For buyer 1 , the difference in valuation between $\{A, B\}$ and $\{A\}$ is equal to 100 . For buyer 2, the difference in valuation between $\{A, B\}$ and $\{B\}$ is equal to 100 .

Case 2: $W \geq 100$.

At the initial time 0 , each buyer $i$ reveals the set of all best response packages $m_{i}$ given by 


$$
m_{1}=\{\phi\} \text { and } m_{2}=\{\phi\} .
$$

The efficient allocations $(\{A\},\{B\}),(\{A, B\}, \phi)$, and $(\phi,\{A, B\})$, the efficient allocation without buyer $2\{A, B\}$, and the efficient allocation without buyer $1\{A, B\}$, which are associated with the profile of representative valuation functions $p$ at the initial time 0 , were not revealed at the initial time 0 . Hence, after the initial time 0 , the auctioneer adjusts the profile of asked price vectors $p=p(t)$ in the following descending manner: at each time $t>0$,

$$
p_{1}(\{A\}\}=p_{1}(\{B\})=p_{2}(\{A\}\}=p_{2}(\{B\})=100-\frac{t}{2},
$$

and

$$
p_{1}(\{A, B\}\}=p_{2}(\{A, B\})=200-t .
$$

Up to the time $100-W$, the buyers continue to reveal the same demand sets as at the initial time 0 . At time $100-W$, where the auctioneer asks the profile of price vectors $p=p(100-W)$ given by

$$
p_{1}(\{A\}\}=p_{1}(\{B\})=p_{2}(\{A\}\}=p_{2}(\{B\})=50+\frac{W}{2},
$$

and

$$
p_{1}(\{A, B\}\}=p_{2}(\{A, B\})=100+W,
$$

each buyer $i$ is willing to reveal the set of all best response packages $m_{i}$ given by

$$
m_{1}=\{\{A, B\}, \phi\} \text { and } m_{2}=\{\{A, B\}, \phi\} .
$$

Note that the efficient allocations $(\{A, B\}, \phi)$ and $(\phi,\{A, B\})$, the efficient allocation without buyer $2\{A, B\}$, and the efficient allocation without buyer $1\{A, B\}$, which are associated with the profile of representative valuation functions $p$ at the initial time 0 , were all revealed at the time $100-W$. Hence, the auctioneer can achieve the correct VCG outcome:

$$
\begin{aligned}
& f(u)=(\{A, B\}, \phi), \\
& x_{1}(u)=p_{2}(\{A, B\})-p_{2}(\phi)=100+W, \text { and } \\
& x_{2}(u)=p_{1}(\{A, B\})-p_{1}(\{A, B\})=0 .
\end{aligned}
$$


The price-demand procedure ends at the time $100-W$, leaking to the public just the following information:

1) Each buyer's valuation for $\{A, B\}$ is equal to $100+W$.

2) Buyer 1's valuations for $\{A\}$ and $\{B\}$, and buyer 2's valuations for $\{A\}$ and $\{B\}$, are all less than $50+\frac{W}{2}$.

Compared with the price-demand procedure at the bottom of Section 6, the above-mentioned price-demand procedure can dramatically save the extent of information leakage. In particular, the auctioneer can perfectly hide the irrelevant information about buyer 1's valuation for item B and the buyer 2' valuation for item A. 


\section{Conclusion}

This paper investigated a general class of open-bid combinatorial auction protocols in the continuous time horizon termed price-demand procedures; the auctioneer asks buyer-dependent price vectors and each buyer reveals demand sets as the set of all best-response packages through time. We assumed the revealed preference activity rule and the connectedness. We demonstrated the characterization result for the class of all price-demand procedures that achieve the VCG outcome. That is, we showed that a price-demand procedure achieves the VCG outcome if and only if the efficient allocation and the efficient allocations without any single buyer, associated with the resultant profile of representative valuation functions, are all revealed in the history. This class has the advantage over the standard practice of the VCG mechanism, because the revelation of best responses through time in the procedure is much easier than the revelation of the entire valuations at one time in the standard practice.

We argued that it is possible for the auctioneer to achieve the VCG outcome in this manner just by making the detail-free contractual agreement with the buyers. In this case, the buyers can easily detect whether the auctioneer succeeds to achieve the correct VCG outcome just by observing the resultant history. Hence, they can leave the selection of price-demand procedure the auctioneer's discretion, without violating the auctioneer's incentive. The auctioneer can make this selection even contingent on his unverifiable private information. He can save irrelevant information leakage to a certain extent by selecting a shortcut procedure. 


\section{References}

Ausubel, L. (2004): “An Efficient Ascending-Bid Auction for Multiple Objects,” American Economic Review 94, 1452-1475.

Ausubel, L. (2006): “An Efficient Dynamic Auction for Heterogeneous Commodities,” American Economic Review 96, 602-629.

Ausubel, L., P. Cramton, and P. Milgrom (2006): “The Clock-Proxy Auction: A Practical Combinatorial Auction Design,” in Combinatorial Auctions, ed. by P. Cramton, Y. Shoham, and R. Steinberg. MIT Press.

Ausubel, L. and P. Milgrom (2006): “The Lovely but Lonely Vickrey Auction,” in Combinatorial Auctions, ed. by P. Cramton, Y. Shoham, and R. Steinberg. MIT Press.

Bikhchandani, S. and J. Ostroy (2002): “The Package Assignment Model,” Journal of Economic Theory 107, 377-406.

Chen, Y. and K. Takeuchi (2010): "Multi-unit Auctions with Package Biddings: An Experimental Comparison of iBEA and Vickrey," Games and Economic Behavior 68, pp.557-569.

Clarke, E. (1971): “Multipart Pricing of Public Goods,” Public Choice 11, 17-33.

Groves, T. (1973): “Incentives in Teams,” Econometrica 41, 617-631.

Gul, F. and E. Stacchetti (2000): “The English Auction with Differentiated Commodities,” Journal of Economic Theory 92, 66-95.

Kelso, A. and V. Crawford (1982): “Job Matching, Coalition Formation, and Gross Substitutes,” Econometrica 50, 1483-1504.

Lahaie, S., F. Constantin, and D. Parkes (2005): "More on the Power of Demand Queries in Combinatorial Auctions: Learning Atomic Languages and Handling Incentives,” Proc. $19^{\text {th }}$ Intern. Joint Conf. Artif. Intell.

Lahaie, S. and D. Parkes (2004): “Applying Learning Algorithms to Preference Elicitation in the Generalized Vickrey Auction,” mimeo, Harvard University.

Matsushima, H. (2011): "Price-Based Combinatorial Auction: Connectedness and Representative Valuations,” Discussion Paper CIRJE-F-806, University of Tokyo. 
Milgrom, P. (2004): Putting Auction Theory to Work, Cambridge University Press: Cambridge.

Mishra, D. and D. Parkes (2007): “Ascending Price Vickrey Auctions for General Valuations,” Journal of Economic Theory 132, 335-366.

Parkes, D. (2006): “Iterative Combinatorial Auctions,” in Combinatorial Auctions, ed. by P. Cramton, Y. Shoham, and R. Steinberg. MIT Press: Cambridge.

Parkes, D. and L. Ungar (2002): “An Ascending-Price Generalized Vickrey Auction,” mimeo, Harvard University.

Rothkopf, M., T. Teisberg, and E. Kahn (1990): “Why Are Vickrey Auctions Rare?” Journal of Political Economy 98, 94-109.

Segal, I. (2006): “Communication Requirements of Combinatorial Allocation Problems,” in Combinatorial Auctions, ed. by P. Cramton, Y. Shoham, and R. Steinberg, MIT Press.

Vickrey, W. (1961): “Counterspeculation, Auctions, and Competitive Sealed Tenders,” Journal of Finance 16, 8-37. 


\section{Appendix A: Proof of Lemma 1}

For every $\varepsilon>0$ and every $u \in U$, we define $u_{i, \varepsilon} \in U_{i}$ by

$$
u_{i, \varepsilon}\left(a_{i}\right)=u_{i}\left(a_{i}\right)+\varepsilon \text { for all } a_{i} \in 2^{L} \backslash\{\phi\} .
$$

Assume that there exists $u \in U$ and $i \in N$ such that

$$
f_{i}(u) \notin A_{i}\left(h_{i}^{T(u)}(u, \gamma)\right) .
$$

Suppose

$$
f_{i}(u)=\phi \notin A_{i}\left(h_{i}^{T(u)}(u, \gamma)\right) .
$$

Then, $h_{i}^{T(u)}(u, \gamma)$ must be consistent with $u_{i, \varepsilon}$ for all $\varepsilon>0$, i.e.,

$$
u_{i, \varepsilon} \in U_{i}\left(h_{i}^{T(u)}(\gamma, T)\right) \text { for all } \varepsilon>0,
$$

which, along with consistency and efficiency, implies that

$$
f\left(u_{i, \varepsilon}, u_{-i}\right)=f(u) \in A^{*}\left(u_{i, \varepsilon}, u_{-i}\right) \text { for all } \varepsilon>0 .
$$

This is a contradiction, because any efficient allocation $a \in A^{*}\left(u_{i, \varepsilon}, u_{-i}\right)$ for $\left(u_{i, \varepsilon}, u_{-i}\right)$ never satisfies $a_{i} \neq \phi$, provided $\varepsilon$ is sufficiently large.

Suppose

$$
f_{i}(u) \neq \phi .
$$

Then, we can select $a_{i} \in 2^{L}$ such that $a_{i} \neq f_{i}(u)$ and $a_{i} \subset f_{i}(u)$, and that for every $a_{i}^{\prime} \in 2^{L}$ satisfying that $a_{i}^{\prime} \notin\left\{a_{i}, f_{i}(u)\right\}$ and $a_{i}^{\prime} \subset f_{i}(u)$,

$$
u_{i}\left(f_{i}(u)\right)-u_{i}\left(a_{i}\right) \leq u_{i}\left(f_{i}(u)\right)-u_{i}\left(a_{i}^{\prime}\right) .^{6}
$$

From (1), we can select $u_{i}^{\prime} \neq u_{i}$ with which $h_{i}^{T(u)}(\gamma, T)$ is consistent, i.e.,

$$
u_{i}^{\prime} \in U_{i}\left(h_{i}^{T(u)}(\gamma, T)\right)
$$

Here, we can select $u_{i}^{\prime}$ and $j \in N \backslash\{i\}$ in a manner that $u_{i}^{\prime}\left(f_{i}(u)\right)-u_{i}^{\prime}\left(a_{i}\right)$ is close enough to zero and

$$
u_{i}^{\prime}\left(f_{i}(u)\right)-u_{i}^{\prime}\left(a_{i}\right)<u_{j}\left(f_{j}(u) \cup f_{i}(u) \backslash a_{i}\right)-u_{j}\left(f_{j}(u)\right) .
$$

\footnotetext{
${ }^{6}$ Note that if $f_{i}(u)$ is a singleton, there exists no such $a_{i}^{\prime}$. Hence, $a_{i}=\phi$ automatically satisfies the requirements.
} 
Let us specify $\hat{a} \in A$ by $\hat{a}_{i}=a_{i}, \quad \hat{a}_{j}=f_{j}(u) \cup f_{i}(u) \backslash a_{i}$, and

$$
\hat{a}_{h}=f_{h}(u) \text { for all } h \in N \backslash\{i, j\} .
$$

From (A-1),

$$
u_{i}^{\prime}\left(f_{i}(u)\right)+\sum_{h \in N \backslash\{i\}} u_{h}\left(f_{h}(u)\right)<u_{i}^{\prime}\left(\hat{a}_{i}\right)+\sum_{h \in N \backslash\{i\}} u_{h}\left(\hat{a}_{h}\right),
$$

implying that $f(u)$ is not efficient for $\left(u_{i}^{\prime}, u_{-i}\right)$. However, since $h_{i}^{T(u)}(u, \lambda)$ is consistent with $u_{i}^{\prime}$, it must hold that $f\left(u_{i}^{\prime}, u_{-i}\right)=f(u)$. This is a contradiction. 


\section{Appendix B: Proof of Lemma 2}

Since $h_{i}^{t}$ is connected, there exists a finite sequence $\left(\tau^{(l)}, a_{i}^{(l)}\right)_{l=1}^{k}$ such that $k \geq 2$, $a_{i}^{(1)}=a_{i}^{\prime}, \quad a_{i}^{(k)}=a_{i}$,

$$
\tau^{(l)} \in[0, t) \text { and } a_{i}^{(l)} \in m_{i}\left(\tau^{(l)}\right) \text { for all } l \in\{1, \ldots, k\},
$$

and

$$
a_{i}^{(l-1)} \in m_{i}\left(\tau^{(l)}\right) \text { for all } l \in\{2, \ldots, k\} .
$$

For every $u_{i} \in U_{i}\left(h_{i}^{t}\right)$ and every $l \in\{2, \ldots, k\}$, since $\left\{a_{i}^{(l)}, a_{i}^{(l-1)}\right\} \subset m_{i}\left(\tau^{(l)}\right)$, it follows

$$
\begin{aligned}
& u_{i}\left(a_{i}^{(l)}\right)-p_{i}\left(\tau^{(l)}\right)\left(a_{i}^{(l)}\right)=u_{i}\left(a_{i}^{(l-1)}\right)-p_{i}\left(\tau^{(l)}\right)\left(a_{i}^{(l-1)}\right), \text { i.e., } \\
& u_{i}\left(a_{i}^{(l)}\right)-u_{i}\left(a_{i}^{(l-1)}\right)=p_{i}\left(\tau^{(l)}\right)\left(a_{i}^{(l)}\right)-p_{i}\left(\tau^{(l)}\right)\left(a_{i}^{(l-1)}\right)
\end{aligned}
$$

Hence,

$$
\begin{aligned}
& u_{i}\left(a_{i}\right)-u_{i}\left(a_{i}^{\prime}\right)=\sum_{l=2}^{k}\left\{u_{i}\left(a_{i}^{(l)}\right)-u_{i}\left(a_{i}^{(l-1)}\right)\right\} \\
& =\sum_{l=2}^{k}\left\{p_{i}\left(\tau^{(l)}\right)\left(a_{i}^{(l)}\right)-p_{i}\left(\tau^{(l)}\right)\left(a_{i}^{(l-1)}\right)\right\} .
\end{aligned}
$$

Let us specify $x_{i}\left(a_{i}, a_{i}^{\prime}, h_{i}^{t}\right) \in R$ as

$$
x_{i}\left(a_{i}, a_{i}^{\prime}, h_{i}^{t}\right)=\sum_{l=2}^{k}\left\{p_{i}\left(\tau^{(l)}\right)\left(a_{i}^{(l)}\right)-p_{i}\left(\tau^{(l)}\right)\left(a_{i}^{(l-1)}\right)\right\} .
$$

Since this specification does not depend on the selection of $u_{i} \in U_{i}\left(h_{i}^{t}\right)$, it follows from (B-1) that for every $u_{i} \in U_{i}\left(h_{i}^{t}\right)$ and every $\left\{a_{i}, a_{i}^{\prime}\right\} \subset A_{i}\left(h_{i}^{t}\right)$,

$$
u_{i}\left(a_{i}\right)-u_{i}\left(a_{i}^{\prime}\right)=x_{i}\left(a_{i}, a_{i}^{\prime}, h_{i}^{t}\right) .
$$




\section{Appendix C: Proof of Proposition 3}

We prove the "if" part as follows. Suppose that for every $h^{t} \in H(\gamma, T)$, there exist $a^{*}\left(h^{t}\right) \in A\left(h^{t}\right)$, and $a^{i *}\left(h_{-i}^{t}\right) \in A^{i}\left(h_{-i}^{t}\right)$ for each $i \in N$, that satisfy (3) and (4) for all $u \in U\left(h^{t}\right)$. Then, we can specify $f: U \rightarrow A$ in a manner that for every $h^{t} \in H(\gamma, T)$ and every $u \in U\left(h^{t}\right)$,

$$
f(u)=a^{*}\left(h^{t}\right) .
$$

We can also specify $x_{i}: U \rightarrow R$ for each $i \in N$ in a manner that for every $h^{t} \in H(\gamma, T)$ and every $u \in U\left(h^{t}\right)$,

$$
x_{i}(u)=\sum_{j \in N \backslash\{i\}} x_{j}\left(a_{j}^{i^{*}}\left(h_{-i}^{t}\right), f_{j}(u), h^{t}\right) .
$$

From Lemma 2 and (4), it follows that

$$
x_{i}(u)=\max _{a^{i} \in A^{i}} \sum_{j \in N \backslash\{i\}} u_{j}\left(a_{j}\right)-\sum_{j \in N \backslash\{i\}} u_{j}\left(f_{j}(u)\right),
$$

which along with (3) implies that the specified mechanism $G=(f, x)$ is VCG.

We prove the "only if" part as follows. Assume that $G=(f, x)$ is VCG and consistent with $(\gamma, T)$. Note from (1) and (2) that for every $i \in N$, every $h_{i}^{t} \in H_{i}^{t}$, and every $\left\{a_{i}, \tilde{a}_{i}\right\} \not \subset A_{i}\left(h_{i}^{t}\right)$, there exists $\left\{u_{i}, \tilde{u}_{i}\right\} \subset U_{i}\left(h_{i}^{t}\right)$ such that

$$
u_{i}\left(a_{i}\right)-u_{i}\left(\tilde{a}_{i}\right) \neq \tilde{u}_{i}\left(a_{i}\right)-\tilde{u}_{i}\left(\tilde{a}_{i}\right) .
$$

Hence, for every $u \in U$, if either $f(u) \notin A\left(h^{T(u)}(u, \lambda)\right)$ or

$$
A^{j}\left(h^{T(u)}(u, \lambda)\right) \cap A^{j^{*}}\left(u_{-j}\right)=\phi \text { for some } j \in N,
$$

then there exist $j \in N$ and $\tilde{u}_{j} \in U_{j}$ such that

$$
\left(\tilde{u}_{j}, u_{-j}\right) \in U\left(h^{T(u)}(u, \lambda)\right),
$$

and for every $i \in N \backslash\{j\}$,

$$
\begin{aligned}
& x_{i}\left(\tilde{u}_{j}, u_{-j}\right)=\max _{a^{i} \in A^{i}}\left\{\tilde{u}_{j}\left(a_{j}\right)+\sum_{h \in N \backslash\{i, j\}} u_{h}\left(a_{h}\right)\right\}-\left\{\tilde{u}_{j}\left(f_{j}(u)\right)+\sum_{h \in N \backslash\{i, j\}} u_{h}\left(f_{h}(u)\right)\right\} \\
& \neq \max _{a^{i} \in A^{i}} \sum_{h \in N \backslash\{i\}} u_{h}\left(a_{h}\right)-\sum_{h \in N \backslash\{i\}} u_{h}\left(f_{h}(u)\right)=x_{i}(u) .
\end{aligned}
$$


This contradicts the supposition that $G$ is consistent with $(\gamma, T)$. Hence, we have proved that for every $u \in U$,

$$
f(u) \in A\left(h^{T(u)}(u, \lambda)\right),
$$

and

$$
A^{j}\left(h^{T(u)}(u, \lambda)\right) \cap A^{j^{*}}\left(u_{-j}\right) \neq \phi \text { for all } j \in N .
$$

Suppose that there exist $\{u, \tilde{u}\} \subset U, \quad j \in N$, and $a^{j} \in A^{j}$ such that

$$
\tilde{u} \in U\left(h^{T(u)}(u, \lambda)\right), \quad a^{j} \in A^{j}\left(h_{-j}^{T(u)}(u, \lambda)\right), \quad a^{j} \in A^{j^{*}}\left(u_{-j}\right),
$$

and

$$
a^{j} \notin A^{j^{*}}\left(\tilde{u}_{-j}\right) .
$$

Without loss of generality, we can select $\tilde{u}$ satisfying that

$$
x_{j}(\tilde{u})=\max _{\tilde{a}^{j} \in A^{j}} \sum_{i \in N \backslash\{j\}} \tilde{u}_{i}\left(\tilde{a}_{i}\right)-\sum_{i \in N \backslash\{j\}} \tilde{u}_{i}\left(f_{i}(u)\right)>\sum_{i \in N \backslash\{j\}} \tilde{u}_{i}\left(a_{i}\right)-\sum_{i \in N \backslash\{j\}} \tilde{u}_{i}\left(f_{i}(u)\right) .
$$

Since

$$
f(u) \in A\left(h^{T(u)}(u, \lambda)\right) \text { and } a^{j} \in A^{j}\left(h_{-j}^{T(u)}(u, \lambda)\right),
$$

it follows that

$$
\sum_{i \in N \backslash\{j\}} \tilde{u}_{i}\left(a_{i}\right)-\sum_{i \in N \backslash\{j\}} \tilde{u}_{i}\left(f_{i}(u)\right)=\sum_{i \in N \backslash\{j\}} u_{i}\left(a_{i}\right)-\sum_{i \in N \backslash\{j\}} u_{i}\left(f_{i}(u)\right)=x_{i}(u),
$$

which implies that $x_{i}(\tilde{u}) \neq x_{i}(u)$. This contradicts the supposition that $G$ is consistent with $(\gamma, T)$. Hence, we have proved that for every $u \in U$ and every $j \in N$,

$$
A^{j}\left(h_{-j}^{T(u)}(u, \lambda)\right) \bigcap\left(\bigcap_{\tilde{u}_{-j} \in U_{-j}\left(h_{-j}^{T(u)}(u, \lambda)\right)} A^{j^{*}}\left(\tilde{u}_{-j}\right)\right) \neq \phi,
$$

which implies that there exists $a^{i^{*}}\left(h_{-i}^{t}\right) \in A^{i}\left(h_{-i}^{t}\right)$ that satisfies (4). Moreover, Lemma 1 implies that there exists $a^{*}\left(h^{t}\right) \in A\left(h^{t}\right)$ for each $i \in N$ satisfying (3).

From the above observations, we have proved the "only if” part. 


\section{Appendix D: Proof of Proposition 4}

The proof of the "if" part is straightforward from the definition of $u_{i}^{\left[h_{i}^{t}\right]}$. From the definition of $u_{i}^{\left[h_{i}^{t}\right]}$ and $a_{i} \in A_{i}\left(h_{i}^{t}\right)$, it follows that if $u_{i} \in U_{i}\left(h_{i}^{t}\right)$, then for every $a_{i}^{\prime} \in A_{i}$,

$$
u_{i}\left(a_{i}\right)-u_{i}\left(a_{i}^{\prime}\right) \geq u_{i}^{\left[h_{i}^{t}\right]}\left(a_{i}\right)-u_{i}^{\left[h_{i}^{t}\right]}\left(a_{i}^{\prime}\right),
$$

and $a_{i}^{\prime} \in A_{i}\left(h_{i}^{t}\right)$ if and only if

$$
u_{i}\left(a_{i}\right)-u_{i}\left(a_{i}^{\prime}\right)=u_{i}^{\left[h_{i}^{t}\right]}\left(a_{i}\right)-u_{i}^{\left[h_{i}^{t}\right]}\left(a_{i}^{\prime}\right)=x_{i}\left(a_{i}, a_{i}^{\prime}, h_{i}^{t}\right),
$$

where we have used the assumption of revealed preference activity rule and Lemma 2.

By letting $a_{i}^{\prime}=\phi$, from $u_{i}(\phi)=u_{i}^{\left[h_{i}^{t}\right]}(\phi)=0$ and $u_{i}\left(a_{i}\right) \geq u_{i}^{\left[h_{i}^{t}\right]}\left(a_{i}\right)$, it follows that $u_{i}\left(a_{i}\right)=u_{i}^{\left[h_{i}^{t}\right]}\left(a_{i}\right)$ if and only if $\phi \in A_{i}\left(h_{i}^{t}\right)$. 


\section{Appendix E: Proof of Theorem 5}

From Proposition 4 and the specification of $u^{\left[h^{t}\right]}$, it follows that for every $i \in N$, every $a_{i} \in A_{i}\left(h_{i}^{t}\right)$, and every $\tilde{a}_{i} \in A_{i}$,

$$
u_{i}^{\left[h_{i}^{t}\right]}\left(a_{i}\right)-u_{i}^{\left[h_{i}^{t}\right]}\left(\tilde{a}_{i}\right) \leq u_{i}\left(a_{i}\right)-u_{i}\left(\tilde{a}_{i}\right) \text { for all } u_{i} \in U_{i}\left(h_{i}^{t}\right) .
$$

Hence, for every $a \in A\left(h^{t}\right)$,

$$
a \in A^{*}(u) \text { for all } u \in U\left(h^{t}\right) \text { if } a \in A^{*}\left(u^{\left[h^{t}\right]}\right) \text {. }
$$

From the specification of $u^{\left[h^{t}\right]}$, it follows that for every $a \in A\left(h^{t}\right)$,

$$
a \in A^{*}\left(u^{\left[h^{t}\right]}\right) \text { if } a \in A^{*}(u) \text { for all } u \in U\left(h^{t}\right) .
$$

Hence, we have proved that for every $a \in A\left(h^{t}\right)$,

$$
a \in A^{*}\left(u^{\left[h^{t}\right]}\right) \text { if and only if } a \in A^{*}(u) \text { for all } u \in U\left(h^{t}\right) \text {. }
$$

This implies that (5) is equivalent to (3). In the same manner, for every $j \in N$ and every $a^{j} \in A^{j}\left(h_{-j}^{t}\right)$,

$$
a^{j} \in A^{j^{*}}\left(u_{-j}^{\left[h^{t}\right]}\right) \text { if and only if } a^{j} \in A^{j^{*}}\left(u_{-j}\right) \text { for all } u_{-j} \in U_{-j}\left(h_{-j}^{t}\right) \text {. }
$$

This implies that (6) is equivalent to (4). 


\section{Appendix F: Proof of Theorem 6}

From (5) and (6), we can select $a^{*}\left(h^{t}\right) \in A\left(h^{t}\right)$, and $a^{i *}\left(h_{-i}^{t}\right) \in A^{i}\left(h_{-i}^{t}\right)$ for each $i \in N$, such that $a^{*}\left(h^{t}\right) \in A\left(h^{t}\right) \cap A^{*}\left(u^{\left[h^{t}\right]}\right)$, and

$$
a^{i *}\left(h_{-i}^{t}\right) \in A^{i}\left(h_{-i}^{t}\right) \cap A^{i *}\left(u_{-i}^{\left[h_{i}^{t}\right]}\right) \text { for all } i \in N .
$$

Hence,

$$
\sum_{i \in N} u_{i}^{\left[h_{i}^{t}\right]}\left(a_{i}^{*}\left(h_{i}^{t}\right)\right) \geq \sum_{i \in N} u_{i}^{\left[h_{i}^{t}\right]}\left(a_{i}\right) \text { for all } a \in A,
$$

and for every $i \in N$,

$$
\sum_{j \in N \backslash\{i\}} u_{j}^{\left[h_{j}^{t}\right]}\left(a_{i}^{j^{*}}\left(h_{i}^{t}\right)\right) \geq \sum_{j \in N \backslash\{i\}} u_{j}^{\left[h_{j}^{t}\right]}\left(a_{j}\right) \text { for all } a^{i} \in A^{i} .
$$

From Proposition 4, $a^{*}\left(h^{t}\right) \in A\left(h^{t}\right)$, and $a^{i^{*}}\left(h_{-i}^{t}\right) \in A^{i}\left(h_{-i}^{t}\right)$, it follows that for every $u \in U\left(h^{t}\right)$ and every $i \in N$,

$$
u_{i}\left(a_{i}^{*}\left(h_{i}^{t}\right)\right)-u_{i}^{\left[h_{i}^{t}\right]}\left(a_{i}^{*}\left(h_{i}^{t}\right)\right) \geq u_{i}\left(a_{i}\right)-u_{i}^{\left[h_{i}^{t}\right]}\left(a_{i}\right) \text { for all } a_{i} \in A_{i},
$$

and for every $j \in N \backslash\{i\}$,

$$
u_{j}\left(a_{j}^{i *}\left(h_{j}^{t}\right)\right)-u_{j}^{\left[h_{j}^{t}\right]}\left(a_{j}^{i *}\left(h_{j}^{t}\right)\right) \geq u_{j}\left(a_{j}\right)-u_{j}^{\left[h_{j}^{t}\right]}\left(a_{j}\right) \text { for all } a_{j} \in A_{j} .
$$

These observations imply that $u^{\left[h^{t}\right]} \in P$ is a universal competitive equilibrium. 